\title{
双相不锈钢各组相循环变形行为的纳米压痕试验 和有限元表征方法研究
}

\author{
郭素娟 ${ }^{1}$ 史艳茹 $^{1}$ 贾云飞 ${ }^{1}$ 赵 剑 $^{2}$ \\ (1. 华东理工大学承压系统与安全教育部重点实验室 上海 200237; \\ 2. 同济大学航空航天与力学学院 上海 200092)
}

摘要: 对双相不锈钢的奥氏体相和铁素体相, 分别开展了不同加载模式(接触载荷和压入位移)和不同加载波形下的单向、循 环纳米压痕试验, 对比分析了两相的基本力学性能和压痕循环变形行为的演化规律。基于压痕试验结果和修正 ABDEL-KARIM-OHNO 非线性随动硬化准则的弹塑性本构模型, 提出一套双相不锈钢奥氏体相和铁素体相的塑性和循环塑 性行为的本构模型参数表征方法。通过对微结构代表性体积单元整体拉伸和循环变形行为进行模拟, 并与宏观试验结果对比, 验证了参数表征方法的合理性。研究结果表明, 铁素体相的强度、硬度和抗楼轮变形的能力均高于奥氏体相, 两相之间通过 晶界产生一定的交互作用; 在接触载荷控制的循环加载条件下, 奥氏体相与铁素体相均产生明显的压痕楼轮现象, 且载荷水 平越高压痕棘轮变形程度越大; 所发展的本构模型参数表征方法可为研究多相材料各组相、小体积材料的循环变形行为提供 借鉴和参考。

关键词：双相不锈钢；纳米压痕；塑性；循环塑性；循环本构参数

中图分类号: TB331

\section{Nanoindentation Experiment and Finite Element Characterization of the Cyclic Deformation Behavior of Duplex Stainless Steel}

\author{
GUO Sujuan $^{1} \quad$ SHI Yanru ${ }^{1} \quad$ JIA Yunfei $^{1} \quad$ ZHAO Jian $^{2}$ \\ (1. Key Laboratory of Pressure System and Safety Ministry of Education, East China University \\ of Science and Technology, Shanghai 200237; \\ 2. School of Aerospace Engineering and Applied Mechanics, Tongji University, Shanghai 200092)
}

\begin{abstract}
The basic mechanical properties and the cyclic indentation deformation behavior of the austenitic and ferrite phases of duplex stainless steel are discussed and compared through the monotonic and cyclic nanoindentation experiments with different loading modes (indentation load and displacement controlled) and different loading waveforms. Based on the indentation experiment results and the elastoplastic constitutive model combined with modified ABDEL-KARIM-OHNO nonlinear dynamic hardening rule, a constitutive model parameter characterization method for the plastic and cyclic plastic behavior of austenite and ferrite phases in duplex stainless steel is developed. The rationality of the parameter characterization method is verified by simulating the overall tensile and cyclic deformation behavior of the representative volume unit of duplex stainless steel, and comparing with the macroscopic experimental results. It is shown that the strength and hardness of the ferrite phase are higher than those of the austenite phase, and the two phases have a certain interaction through the grain boundary. Both the austenite phase and the ferrite phase exhibit obvious indentation ratcheting phenomenon under cyclic indentation loadings, while the ratcheting deformation increases with loading levels. The developed constitutive model parameter characterization method can provide reference for studying the cyclic deformation behavior of each phase of multiphase materials and small-volume materials.
\end{abstract}

Key words: duplex stainless steel; nano-indentation; plasticity; cyclic plasticity; parameters of cyclic constitutive model

* 国家自然科学基金(11872181)、上海市自然科学基金(20ZR1462600, 18ZR1408900)和航空科学基金(201909038001)资助项目。20200616 收到 初稿, 20200911 收到修改稿 


\section{0 前言}

双相不锈钢综合了铁素体相和奥氏体相的性能 优点, 不仅具有较高的强度、韧性和耐腐蚀性, 还 具有良好的导热性和可焊性, 因而被广泛应用于石 油化工、能源工程等领域 ${ }^{[1]}$ 。在工程应用中，构件 由于受到扰动载荷、开机启动-停机休整等因素的影 响, 往往承受循环载荷的作用。在循环载荷作用下, 双相不锈钢构件由于各组相几何和力学性能不连 续, 其内部容易产生晶粒尺度的应力集中, 在较小 的宏观载荷下，其内部就可能产生较大的局部塑性 变形 ${ }^{[2]}$, 进而诱发早期的疲劳裂纹萌生。因此, 充 分了解和准确标定双相不锈钢各组相的微观力学性 能, 同时研究其各组相的局部变形及演化特征, 并 对其进行合理描述, 将会为进一步深入研究材料构 件在循环载荷下的疲劳损伤机理提供微观依据, 对 保证材料构件的长周期安全服役具有重要意义 ${ }^{[3]}$ 。

研究表明 ${ }^{[4]}$, 材料在微观尺度下往往表现出与宏 观条件完全不同的特性, 因此有必要借助微观力学研 究方法对双相材料内部各相的力学性能和循环演化特 征进行研究。纳米压痕法 ${ }^{[5]}$ 作为一种常用的微观试验 方法, 已被广泛用于材料局部力学性能表征, 如材料 的弹性模量、硬度以及材料在微纳米尺度上的局部变 形行为 ${ }^{[6]}$ 等。基于循环纳米压痕技术, 谢季佳等 ${ }^{[7]}$ 研究 了纳米晶 $\mathrm{Ni}$ 和粗晶 $\mathrm{Ni}$ 在纳米尺度下的循环变形行为; JIA 等 ${ }^{[8-9]}$ 通过循环压痕试验, 分别观察了牙釉质和铝 在循环接触载荷下的压入深度演化规律; CUI 等 ${ }^{[10]}$ 研 究了双相不锈钢奥氏体相和铁素体相中心的循环压痕 行为; 孔祥霞等 ${ }^{[11]}$ 通过纳米压痕试验, 比较了 $\mathrm{Cu} / \mathrm{SAC} 0705 / \mathrm{Cu}$ 微焊点在不同最大载荷下的蠕变性 能; 徐连勇等 ${ }^{[5]}$ 则采用恒加载速率/载荷法在室温下对 复合钎料的压痕变形行为和屈服台阶出现机制进行了 分析。然而, 国内外关于双相不锈钢各组相力学性能 的对比研究还较少, 缺乏对奥氏体相和铁素体相循环 压痕作用下变形行为的系统研究。为更深入地分析双 相不锈钢各组相的交互作用机理，基于微观测试结果 对微观组相的基本力学性能和循环变形参数进行标定 十分必要。一些学者通过纳米压痕试验结果获取材料 的塑性相关参数 ${ }^{[12-14]}$, 如 DAO 等 ${ }^{[12]}$ 通过对 76 种金属 材料的纳米压痕试验数据进行量纲分析, 得到了通过 材料压痕数据确定其弹塑性特征参数的关系式; 马永 等 ${ }^{[14]}$ 利用有限元数值模拟方法对单向纳米压痕过程进 行反演分析, 发展了确定金属材料屈服极限和应变强 化指数的相关方法。然而, 上述方法的材料通用性还
有待进一步发展和验证; 基于纳米压痕试验, 获取材 料循环变形参数的方法还未见报道, 亟需深入研究和 发展。

基于上述研究背景和现状, 本文首先针对某双相 不锈钢中的奥氏体相和铁素体相, 分别开展了位移和 接触载荷控制模式下的单向和循环纳米压痕试验, 对 比和探究了奥氏体相和铁素体相的基本力学性能和 压痕循环变形特征; 其次, 基于压痕试验结果, 结合 基于修正 ABDEL-KARIM-OHNO 非线性随动硬化准 则的弹塑性本构模型，通过有限元反演对比分析方 法, 发展了一套与该本构模型相配套的双相不锈钢奥 氏体相和铁素体相的塑性和循环塑性行为的参数表 征方法, 并通过与相应试验结果对比, 验证了表征方 法的合理性。研究成果对获取和描述多相材料各组 相、小体积材料的循环变形行为具有重要意义。

\section{1 试验方法}

\section{1 各组相纳米压痕试验}

本文研究对象为某商用双相不锈钢 (奥氏体相 占比约 60\%)。为讨论双相不锈钢中奥氏体相和铁素 体相的基本力学性能及压痕循环变形行为的演化规 律及差异，并对其循环塑性行为进行表征，首先分 别对奥氏体相和铁素体相开展压入位移和接触载荷 控制的单向、循环压痕试验。压痕试验在 Nano Indenter G200 纳米压痕仪上进行, 通过线切割获取 一块 $20 \mathrm{~mm} \times 20 \mathrm{~mm} \times 8 \mathrm{~mm}$ 的双相不锈钢样胚, 通 过手工打磨、机械抛光及在浓度为 $20 \% \mathrm{NaOH}$ 溶液 中轻微电解腐蚀后, 得到可在光学显微镜下观察到 清晰晶界的光滑镜面试样。在显微镜下, 双相不锈 钢呈现出图 1 所示的微观组织图, 图 1 中突起的区 域为奥氏体相 $(\mathrm{A})$, 下凹区域为铁素体相 $(\mathrm{F})$ 。在此 处及后文的图、表中，均用 $\mathrm{A}$ 表示奥氏体相，F 表 示铁素体相。试验选取 XP Berkovich 压头, 在室温 下进行。单向压痕试验采用压入位移控制模式, 取 试验峰值位移为 $1500 \mathrm{~nm}$, 热漂 $0.05 \mathrm{~nm} / \mathrm{s}$; 循环 压痕则采用接触载荷控制模式，考虑如图 $2 a 、 2 b$ 所示的增幅和等幅两种加载波形, 热漂 $0.1 \mathrm{~nm} / \mathrm{s}$ 。 图 2a 的增幅加载波形, 取峰值载荷 $200 \mathrm{mN}$, 分五 个增量步进行加卸载, 逐步增加到最大载荷, 且分 别在每个增量步的峰值载荷设置保载时间为 $10 \mathrm{~s}$ 或 $20 \mathrm{~s}$ 。图 $2 \mathrm{~b}$ 所示的等幅加载波形, 分别取峰值接触 载荷 $F_{\max }$ 为 $50 \mathrm{mN} 、 100 \mathrm{mN}$ 和 $200 \mathrm{mN}$, 谷值接触 载荷 $F_{\mathrm{min}}$ 为 $5 \mathrm{mN}$, 加载速度为 $10 \mathrm{mN} / \mathrm{s}$, 循环周次 为 30 周。为减小试验分散性和晶界的影响, 每个载 
荷工况均在晶粒中心远离晶界的位置重复 6 次试验。 虽然 6 次试验的结果均比较接近, 但为保证对比的精 确程度, 取 6 次的平均值作为最终试验结果。

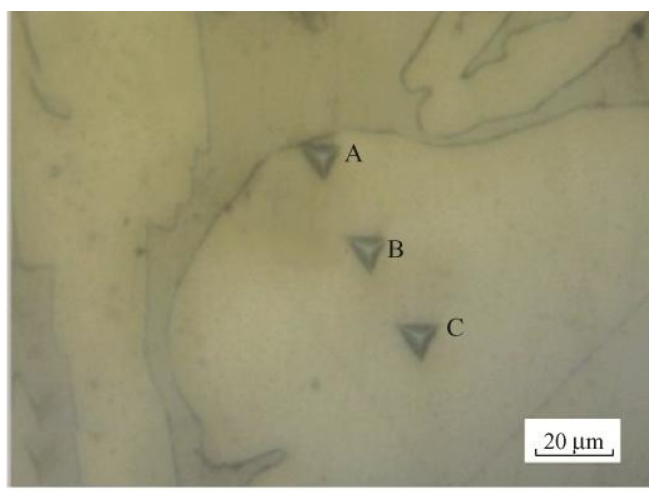

(a) 奥氏体

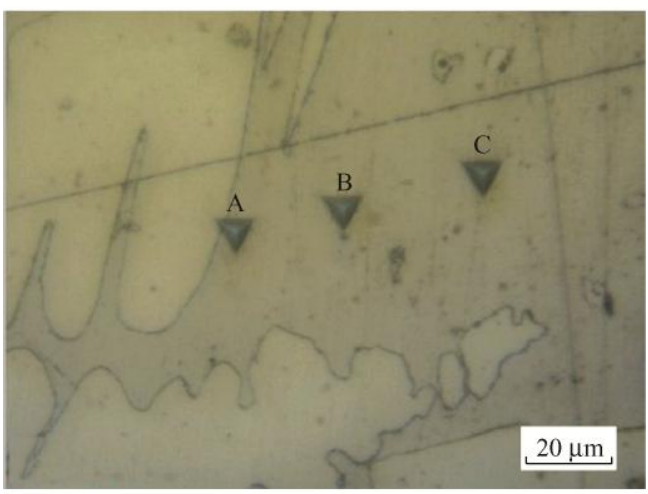

(b) 铁素体

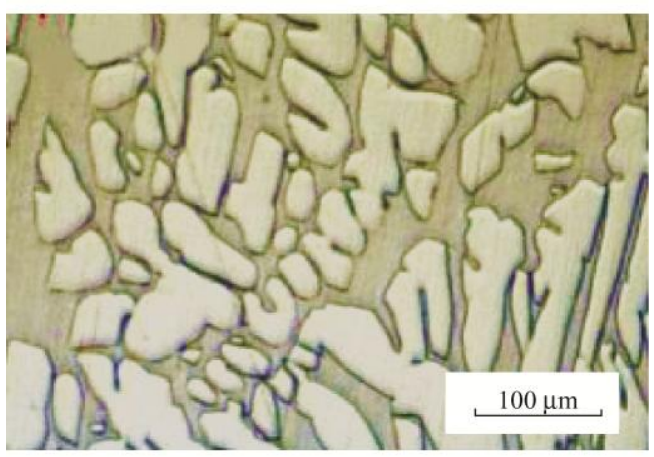

(c) 微观组织

图 1 双相不锈钢的微观组织图及单向压痕点

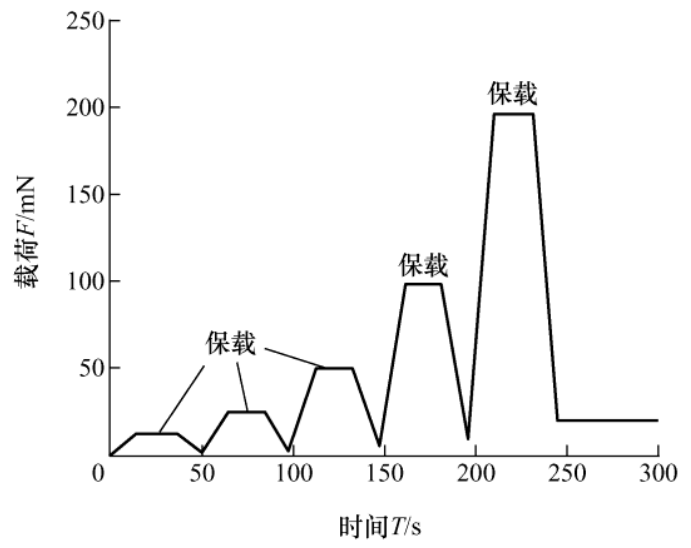

(a) 增幅加载

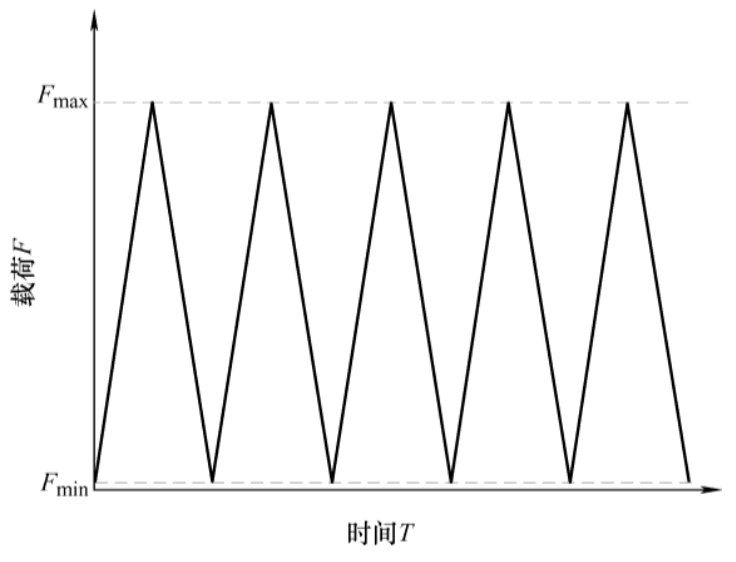

(b) 等幅加载

图 2 循环压痕试验加载波形

\section{2 双相不锈钢单轴拉伸和循环变形试验}

为进一步验证后文中奥氏体相和铁素体相塑性 和循环塑性参数表征方法的合理性, 本节增加双相 不锈钢的室温宏观单轴拉伸和循环变形试验, 为后 文提供对比依据。宏观拉伸试验在原位拉伸仪上进 行, 试验采用如图 3 所示的原位拉伸试样, 取应变 速率为 $0.01 \% / \mathrm{s}$ 。循环变形试验在 INSTRON 电液伺 服疲劳试验机上进行, 试验采用应力控制模式, 试 样为图 4 所示的圆棒试样。应力控制的循环变形试 验考虑平均应力为 $30 \mathrm{MPa}$, 应力幅值分别 $450 \mathrm{MPa}$ 和 $500 \mathrm{MPa}$, 加载速率为 $200 \mathrm{MPa} / \mathrm{s}$, 试验周次取 40 周。单轴拉伸和循环变形试验结果将在后文中验 证模型参数时一并给出。

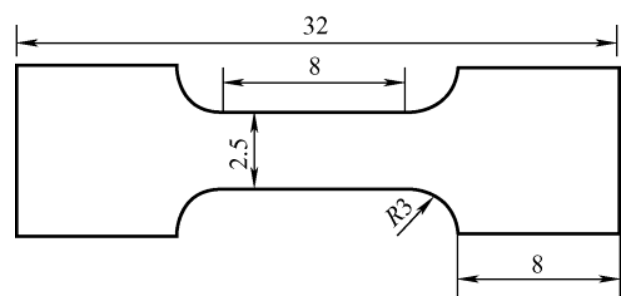

图 3 单轴拉伸试验所用试样尺寸示意图(mm)

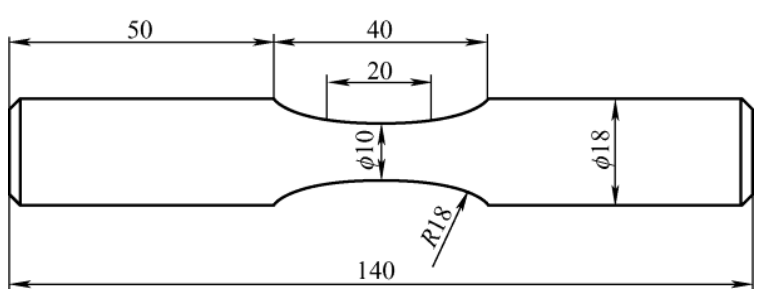

图 4 循环变形试验所用试样尺寸示意图 $(\mathrm{mm})$

在进行双相不锈钢的纳米压痕和宏观试验研究 时, 并未考虑相变和孪晶现象。这是因为该双相不 
锈钢中奥氏体相有如下特点 ${ }^{[10]}$ : (1) 较高的堆垛层 错能(Stacking fault energy, SFE)水平, 本文研究的 奥氏体相在室温下的 SFE 值约为 $57.76 \mathrm{~mJ} / \mathrm{m}^{2[10]}$, 已有研究表明在 SFE 水平较高 $\left(>50 \mathrm{~mJ} / \mathrm{m}^{2}\right.$ ) 时, 马氏 体相变和机械孪晶均受到抑制, 位错演化是主要的 变形机制 ${ }^{[15-16]}$; (2) 承受较高的加载速率, 在试验过 程中由于绝热增温, 试样温度随加载速率的增加而 升高 ${ }^{[17]}$, 温度的升高会进一步导致 SFE 值升高, 使 奥氏体相更稳定。而本研究的加载速率高于相关文 献中的加载速率, 故本文试验过程中奥氏体相更加 稳定。此外, 文献[10]对同样材料进行循环纳米压 痕试验下透射电子显微镜 (Transmission electron microscopy, TEM) 的观察结果也显示, 奥氏体变 形只有位错这一种机制, 未发现相变和孪晶。综上 所述, 可认为本文使用的双相不锈钢中奥氏体相并 未发生相变和孪晶, 因此在相关试验和后文的模拟 计算中不考虑这两种微结构演化现象。

\section{2 试验结果与分析}

\section{1 双相不锈钢各组相的基本力学性能}

\subsection{1 晶界对各组相局部力学性能的影响}

双相不锈钢由奥氏体相和铁素体相组成, 两 相力学性能不同, 通过晶界相联系。为讨论两相 间的交互作用和晶界对奥氏体相与铁素体相局部 力学性能的影响, 本文首先选取有代表性的奥氏 体晶粒和铁素体晶粒, 在其内部与晶界距离不 同的地方选取合适的位置 (晶粒中心与同侧晶 界之间), 如图 1 所示, 进行压入载荷控制下的 单向压痕试验。最终得到奥氏体与晶界距离分别 为 $8 \mu \mathrm{m} 、 30 \mu \mathrm{m}$ 和 $58 \mu \mathrm{m}$ 的几个位置点(在图 $1 \mathrm{a}$ 中分别标记为 $A 、 B 、 C)$, 铁素体相与晶界距离 分别为 $5 \mu \mathrm{m} 、 32 \mu \mathrm{m}$ 和 $64 \mu \mathrm{m}$ 的几个位置点(在图 $1 \mathrm{~b}$ 中分别标记为 $\mathrm{A} 、 \mathrm{~B} 、 \mathrm{C}$ ), 对其进行单向纳米 压痕试验。图 $5 \mathrm{a}$ 和图 $5 \mathrm{~b}$ 分别给出了奥氏体相和 铁素体相上在上述不同位置压痕点处的接触载荷压入深度演化曲线。可以看出, 奥氏体相中随着 试验位置与晶界距离的增加, 相同最大接触载荷 对应的最大压入深度也逐渐增加, 这说明奥氏体 中距离晶界越远, 强度越弱; 在铁素体相中随着 试验位置与晶界距离的增加, 相同最大接触载荷 对应的最大压入深度逐渐减小, 这说明铁素体中 距离晶界越远, 强度越强。另外, 观察还发现两 相各点的压入深度变化与其到晶界的距离并不呈正
比关系：随着距离增加, 压入深度之间的差值逐 渐变小, 晶界对两相组织只在一定的距离范围内 有影响, 当超过一定距离后影响可以忽略。在后 续试验中, 为避免晶界对试验结果的影响, 均选 择各相中远离晶界的位置进行试验。

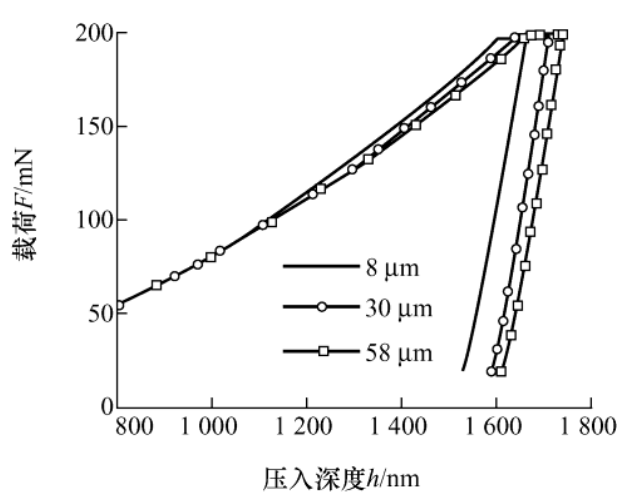

(a) 奥氏体

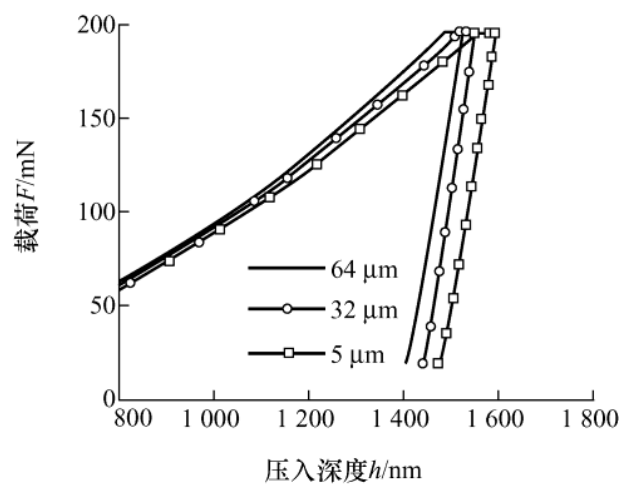

(b) 铁素体

图 5 奥氏体相和铁素体相在不同压痕位置 的载荷-压入深度曲线

\subsection{2 奥氏体相和铁素体相的基本力学性能分析}

在压入位移控制模式下, 取晶粒中心远离界 面的位置, 设置最大压入深度为 $1500 \mathrm{~nm}$, 应变 速率恒定为 $0.05 / \mathrm{s}$, 分别对奥氏体相和铁素体相 重复进行单向压痕试验, 获取两相的基本力学性 能。基于 OLIVER-PHARR 方法 ${ }^{[10]}$ 得到的奥氏体 相 (A) 和铁素体相 $(\mathrm{F})$ 各次试验的弹性模量、硬度 值及其平均值如表 1 所示。表 1 中, $E$ 为弹性模 量, $E_{\mathrm{m}}$ 为弹性模量的平均值, $H$ 为硬度, $H_{\mathrm{m}}$ 为 硬度的平均值, $F_{\max }$ 为压入载荷的最大值, $F_{\mathrm{m}}$ 为 压入载荷最大值的平均值。从表 1 中可以看出, 奥氏体相的平均弹性模量约为 $224.8 \mathrm{GPa}$, 平均硬 度为 $5.8 \mathrm{GPa}$; 铁素体相的平均弹性模量约为 $251.7 \mathrm{GPa}$, 平均硬度约为 $6.2 \mathrm{GPa}$, 铁素体的硬度 和弹性模量均高于奥氏体。 
表 1 两相的试验结果

\begin{tabular}{ccccccccc}
\hline 序号 & $\begin{array}{c}\text { 弹性模量 } \\
E / \mathrm{GPa}\end{array}$ & $\begin{array}{c}\text { 弹性模量均值 } \\
E_{\mathrm{m}} / \mathrm{GPa}\end{array}$ & $\begin{array}{c}\text { 分散系数 } \\
\delta_{1}(\%)\end{array}$ & 硬度 $H / \mathrm{GPa}$ & $\begin{array}{c}\text { 硬度均值 } \\
H_{\mathrm{m}} / \mathrm{GPa}\end{array}$ & $\begin{array}{c}\text { 分散系数 } \\
\delta_{2}(\%)\end{array}$ & $\begin{array}{c}\text { 压入载荷最大值压入载荷最大 } \\
F_{\text {max }} / \mathrm{mN}\end{array}$ & $\begin{array}{c}\text { 分散系数 } \\
F_{\mathrm{m}} / \mathrm{mN}\end{array}$ \\
\hline $\mathrm{\delta}_{3}(\%)$
\end{tabular}

在相同压入深度下, 铁素体的接触载荷高于奥 氏体的接触载荷。为讨论不同奥氏体或铁素体晶粒 试验结果的重复性, 本文针对 6 次重复试验的结果 定义了分散系数, 如式(1)所示

$$
\delta=\left|\alpha-\alpha_{m}\right| / \alpha_{m} \times 100 \%
$$

式中, $\delta$ 表示测量值的分散系数; $\alpha$ 表示试验测量 值; $\alpha_{m}$ 表示 6 次试验测量的平均值。表 1 中 $\delta_{1}$ 为弹 性模量的分散系数, $\delta_{2}$ 为硬度的分散系数, $\delta_{3}$ 为压 入载荷最大值的分散系数。从表 1 中各次试验的分 散系数可看出, 在奥氏体相和铁素体相中远离晶界 的位置, 弹性模量、硬度和最大接触载荷的重复性 非常好。

\section{2 双相不锈钢各组相的压痕棘轮行为}

本节通过接触载荷控制的循环压痕试验, 研究 奥氏体相和铁素体相的局部塑性循环变形行为。试 验选取图 $2 b$ 所示的等幅循环加载波形, 为讨论载荷 水平的影响, 加载峰值载荷分别定为 $50 \mathrm{mN}$ 、 $100 \mathrm{mN}$ 和 $200 \mathrm{mN}$, 谷值载荷为 $5 \mathrm{mN}$, 试验过程中 加载速率为 $10 \mathrm{mN} / \mathrm{s}$, 循环周次为 30 周。图 $6 \mathrm{a}$ 和 $6 \mathrm{c}$ 分别给出了上述三个载荷水平下, 奥氏体相(A) 和铁素体相 $(\mathrm{F})$ 的循环载荷-压入深度曲线和最大压 入深度随循环周次的演化曲线, 可以看出: (1) 在接 触载荷控制的等幅循环载荷下, 奥氏体相和铁素体 相各周次的最大压入深度随着循环周次的增加而增 加, 呈现出明显的压痕棘轮现象; (2) 在相同的载荷 水平下，铁素体相的压入深度小于奥氏体相的压入 深度, 经过 30 个周次, 铁素体相最大压入深度增量 小于奥氏体相; (3) 无论是奥氏体相还是铁素体相,
载荷水平越高, 其各周次的最大压入深度越大, 且 在各载荷水平下, 两相前 3 5 周内压入深度增长很 快, 在随后的周次中逐步减小。图 7 给出了奥氏体 相 $(\mathrm{A})$ 和铁素体相 $(\mathrm{F})$ 在不同载荷水平下每个周次的 压入深度增量的柱状图, 可见在不同载荷水平下, 奥氏体相和铁素体相每个周次的压入深度增量(压 痕棘轮增量)随着循环周次的增加快速减小, 最大值 集中在前 $5 \sim 10$ 个周次, 之后缓慢趋于零; 载荷水 平越高, 每周压入深度增量越大, 且在相同的载荷 水平下铁素体的压入深度增量远低于奥氏体的增 量, 这表明铁素体的抗棘轮变形能力大于奥氏体。

\section{3 各组相循环塑性行为的描述和表征}

从微观层面, 双相不锈钢受奥氏体和铁素体相 几何和力学性能不连续等微观因素的影响, 极易 发生局部应力集中, 在较低的宏观载荷下, 就可 能产生局部的裂纹形核和破坏。因此, 发展合理 的模拟表征方法, 获取双相不锈钢各组相的塑性 和循环塑性性能和相关参数, 将为进一步模拟分 析材料内部的局部应力、应变分布和损伤演化规 律提供必要的基础数据。为此, 本部分基于有限 元反演和分析, 结合基于修正的 ABDEL-KARIMOHNO 非线性随动硬化演化准则的弹塑性本构模 型, 发展了一套与之相对应的获取双相不锈钢中 奥氏体相和铁素体相塑性和循环塑性参数的数值 表征方法, 并与相应纳米压痕试验对比, 验证了 方法和参数的合理性。 


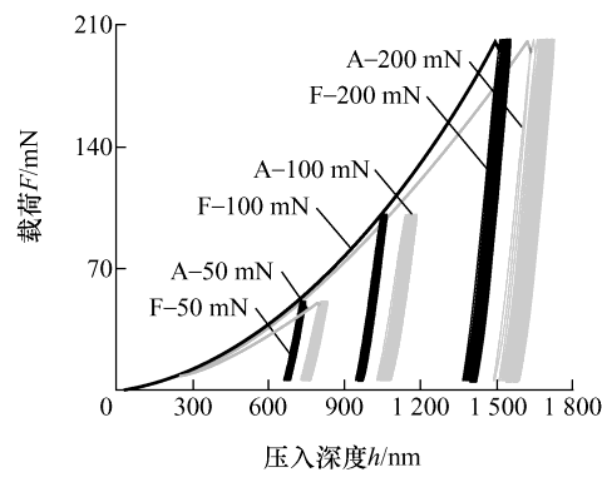

(a) 试验载荷-压入深度曲线

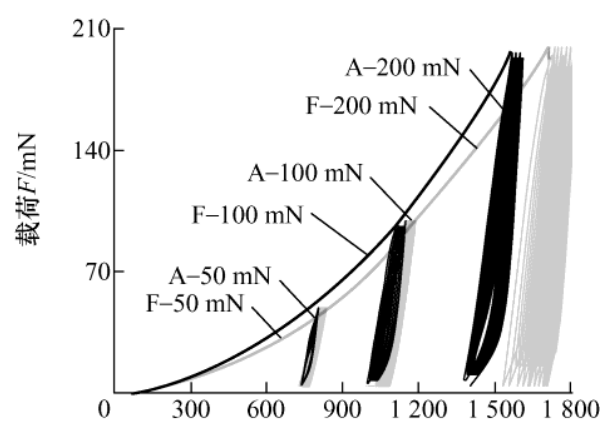

压入深度 $h / \mathrm{nm}$

(b) 有限元模拟载荷-压入深度曲线

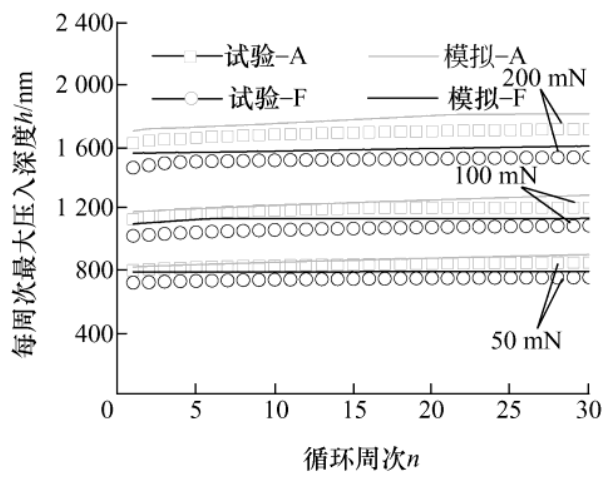

(c) 最大压入深度随环周次的演化曲线

图 6 两相等幅循环载荷下的循环变形行为

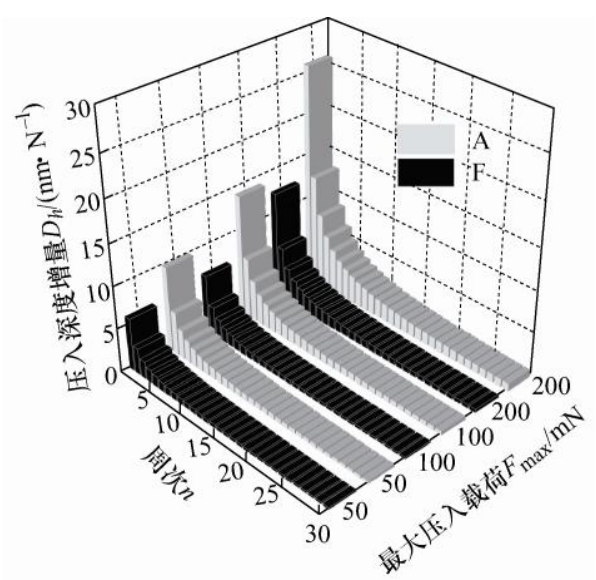

图 7 奥氏体相和铁素体相在等幅循环载荷下 每个周次的压入深度增量

\section{1 循环弹塑性本构模型}

为合理描述双相不锈钢各组相的塑性和循环塑 性行为，本文选取以下循环塑性本构模型对奥氏体 和铁素体相的单向压痕和循环压痕行为进行有限元 模拟和反演，最终通过与试验对比表征相应的塑性 和循环塑性参数。循环弹塑性模型的主控方程为

$$
\begin{aligned}
& \boldsymbol{\varepsilon}=\boldsymbol{\varepsilon}^{e}+\boldsymbol{\varepsilon}^{p} \\
& \varepsilon^{e}=D^{-1}: \sigma \\
& \dot{\boldsymbol{\varepsilon}}^{p}=\sqrt{\frac{3}{2}} \lambda \frac{\boldsymbol{s}-\boldsymbol{\alpha}}{\|\boldsymbol{s}-\boldsymbol{\alpha}\|} \\
& F_{y}=\frac{3}{2}(s-\alpha):(s-\alpha)-Q \\
& \dot{p}=\left(\frac{3}{2} \dot{\boldsymbol{\varepsilon}}^{p}: \dot{\boldsymbol{\varepsilon}}^{p}\right)^{1 / 2}
\end{aligned}
$$

式中, $\varepsilon 、 \varepsilon^{p} 、 \varepsilon^{e}$ 和 $\dot{\varepsilon}^{p}$ 分别表示总应变、非弹性应 变、弹性应变和非弹性应变率的二阶张量； $\boldsymbol{D}$ 为弹 性刚度矩阵; $s$ 和 $\alpha$ 为偏应力和偏背应力张量; $Q$ 为 各向同性抗力; $F_{y}$ 为 VON-MISES 屈服函数; $\lambda$ 为 塑性因子，可以由一致性条件定出; $\dot{p}$ 为非弹性累 积塑性应变率。

其中，随动硬化律采用修正的 ABDELKARIM-OHNO 非线性演化方程

$$
\begin{gathered}
\alpha=\sum_{i=1}^{M} \boldsymbol{\alpha}_{i} \\
\dot{\boldsymbol{\alpha}}_{i}=\zeta_{i}\left[\frac{2}{3} r_{i} \dot{\varepsilon}^{p}-\mu \boldsymbol{\alpha}_{i} \dot{p}-H\left(f_{i}\right) \boldsymbol{\alpha}_{i}(1-\mu) \dot{p}\right] \\
\boldsymbol{K}_{i}=\frac{\boldsymbol{\alpha}_{i}}{\left\|\boldsymbol{\alpha}_{i}\right\|} \\
f_{i}=\left\|\boldsymbol{\alpha}_{i}\right\|^{2}-r_{i}^{2}=0
\end{gathered}
$$

式中, $\zeta_{i} 、 r_{i}$ 为材料参数, 可以简单由单调拉伸试 验结果得到; $\mu_{i}$ 为棘轮系数; $f_{i}$ 为临界面; $H(x)$ 为 HEAVISIDE 函数。

各向同性硬化律采用如下带有动态回复项的各 向同性演化方程

$$
\dot{Q}=b\left(Q_{s a}-Q\right) \dot{p}
$$

式中, $Q_{s a}$ 为饱和各向同性变形抗力; $Q$ 为各向同性 变形抗力; $b$ 为与温度相关的材料参数, 反映各向 同性变形抗力 $Q$ 的演化速度; $p$ 为累积塑性应变。 后文中将通过对单向和循环纳米压痕试验的模拟, 确定和表征上述循环塑性本构模型中涉及的塑性和 循环塑性材料参数。 


\section{2 奥氏体和铁素体相的拉伸曲线反演和获取}

第 3.1 节中循环塑性本构模型的部分材料参数, 需要通过材料的拉伸应力-应变曲线获得。本节首先 选取表 1 中针对奥氏体相和铁素体相的单向纳米压 痕试验中接近平均值的试验结果作为参照和输入, 然后结合 DAO 等 ${ }^{[12]}$ 所建立的量纲函数和有限元反 演对比分析方法, 获取双相不锈钢中奥氏体相和铁 素体相符合式(12)的单轴幂强化弹塑性拉伸应力-应 变关系

$$
\begin{gathered}
\sigma= \begin{cases}E \varepsilon & \sigma \leqslant Q_{0} \\
Q_{0}\left(1+\frac{E}{Q_{0}} \varepsilon_{p}\right)^{n} & \sigma>Q_{0}\end{cases} \\
\varepsilon=\varepsilon_{y}+\varepsilon_{p} \\
\sigma_{y}=E \varepsilon_{y}
\end{gathered}
$$

式中, $E$ 为弹性模量; $n$ 为幂强化指数; $Q_{0}$ 为初始 屈服应力; $\varepsilon_{y}$ 为与区服应力相对应的相关应变; $\varepsilon_{p}$ 为总应变减去 $\varepsilon_{y}$ 。有限元反演主要基于量纲分析法 获得的初步参数, 通过单向纳米压痕过程的模拟和 试验结果的对比, 对其进一步修正, 最终确定相关 材料参数。

有限元反演采用图 8 所示的轴对称有限元模 型, 模型将 BERKOVICH 压头等效为 $140.6^{\circ}$ 的圆雉 压头, 建立材料和压头的接触关系。模拟时在模型 底部施加 $Y$ 轴方向约束, 左侧对称轴上施加了 $X$ 轴方向对称约束, 对与压头绑定的参考点施加位移 或载荷。最终, 通过优化对比和反演计算得到奥 氏体的幂强化指数 $n=0.27$, 初始屈服应力 $Q_{0}=358.2 \mathrm{MPa}$; 铁素体的幂强化指数 $n=0.3$, 初始 屈服应力 $Q_{0}=381.2 \mathrm{MPa}$, 与上述参数相对应的两相 拉伸应力-应变曲线如图 9 所示。为验证上述参数及 其所确定的奥氏体相和铁素体相拉伸曲线的合理 性, 图 10 给出基于上述参数进行单向压痕模拟所得 的接触载荷-压入深度曲线与试验结果的对比。可以 看出, 模拟结果与试验吻合良好。为进一步说明模 拟结果和试验结果之间的符合程度, 此处引入误差 $R$, 计算公式为

$$
R=\frac{\left|x_{i}-x_{i 0}\right|}{x_{i 0}} \times 100 \%
$$

式中， $x_{i} 、 x_{i 0}$ 分别表示模拟值和试验值。根据式(15) 计算图 10 中最大压入载荷模拟值与试验值的误差, 可得奥氏体为 $3.5 \%$ ，铁素体为 $0.3 \%$ 。可见本节通 过量纲分析和有限元反演优化所确定的奥氏体相和
铁素体相的力学性能参数合理可用。此外, 需要强 调的是, 此处和后文中对奥氏体相进行单向和循环 压痕有限元模拟时, 均采用图 8 所示的有限元模型。

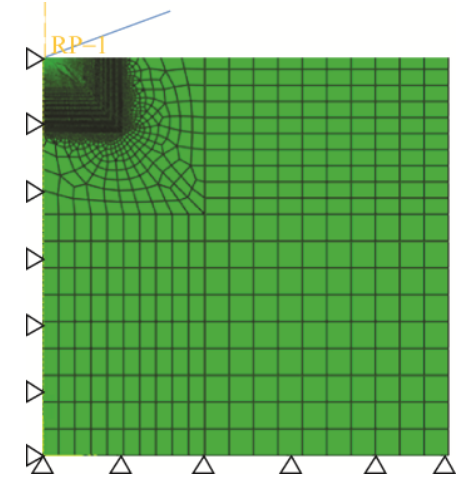

图 8 有限元模型与网格划分

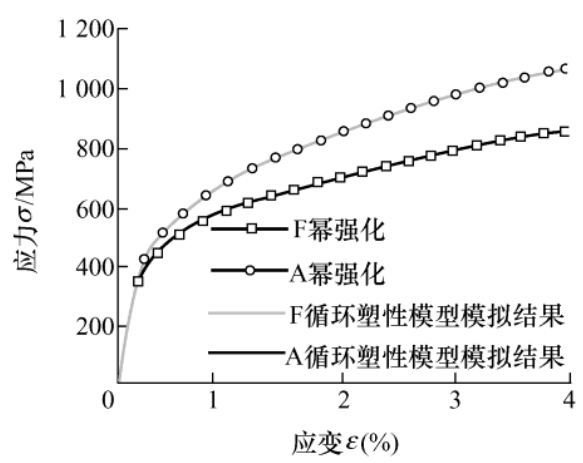

图 9 幂强化关系计算和循环塑性本构模型模拟的 奥氏体相和铁素体相拉伸曲线对比

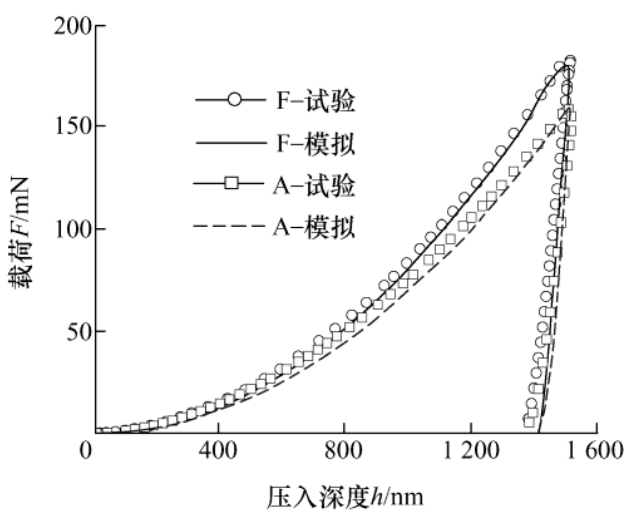

图 10 奥氏体相和铁素体相的试验和有限元模拟的 接触载荷-压入深度对比曲线

\section{3 循环塑性本构模型的参数确定及验证}

\subsection{1 参数 $\zeta_{i}$ 和 $r_{i}$ 的初步确定}

参数 $\zeta_{i}$ 和 $r_{i}$ 是与随动硬化有关的参数。这两组 参数的确定可由奥氏体相和铁素体相的拉伸曲线获 得 ${ }^{[18]}$ 。根据拉伸应力-应变曲线取出应力-塑性应变 曲线, 在该曲线上取若干个点, 采用式(16)和式(17) 确定 $\zeta_{i}$ 和 $r_{i}$ 的值, 考虑计算精度, 本文取 $M=11$ 。 


$$
\zeta_{k}=\frac{1}{\varepsilon_{k}^{p}} \quad k=1,2, \cdots, M
$$

$\gamma_{k}=\left(\frac{\sigma_{k}-\sigma_{(k-1)}}{\varepsilon_{k}^{p}-\varepsilon_{k-1}^{p}}-\frac{\sigma_{(k+1)}-\sigma_{k}}{\varepsilon_{k+1}^{p}-\varepsilon_{k}^{p}}\right) \varepsilon_{k}^{p} \quad k=1,2, \cdots, M$

式中, 当 $k=1$ 时, $\sigma_{0}$ 是曲线 $\varepsilon_{p}=0$ 时的 $\sigma$ 值。

\subsection{2 与非线性各向同性相关的参数确定}

在确定随动硬化参数之后加入各向同性硬化参 数, 进行循环压痕的反演和比较, 更新各向同性演 化参数时, 一并更新随动硬化参数, 以去掉各向同 性硬化的影响。最终保证使用确定参数模拟所得的 奥氏体相和铁素体相的单拉曲线、单向压痕曲线和 循环纳米压痕曲线三者均与试验结果吻合。根据上 述参数标定方法, 第 3.1 节所介绍的弹塑性循环本 构模型涉及的所有塑性和循环塑性参数见表 2 。

\section{表 2 本构参数值}

\begin{tabular}{|c|c|}
\hline 参数类别 & 材料参数值 \\
\hline $\begin{array}{l}\text { 基本参数 } \\
\text { (A) }\end{array}$ & $M=11, E=224.8 \mathrm{GPa}, v=0.33, Q_{0}=368.2 \mathrm{MPa}$ \\
\hline $\begin{array}{c}\text { 随动硬化参 } \\
\text { 数(A) }\end{array}$ & $\begin{array}{l}\zeta_{1}=621.6, \zeta_{2}=193.6, \zeta_{3}=93.0, \zeta_{4}=60.8, \zeta_{5}=38.3 \\
\zeta_{6}=27.9, \zeta_{7}=21.0, \zeta_{8}=17.4, \zeta_{9}=14.1 \zeta_{10}=10.3, \zeta_{11}=4.0 \\
r_{1}=42.7, r_{2}=59.1, r_{3}=53.9, r_{4}=49.3, r_{5}=51.9, r_{6}=44.6 \\
r_{7}=38.6, r_{8}=34.9, r_{9}=48.2, r_{10}=66.0, r_{11}=750.7\end{array}$ \\
\hline $\begin{array}{c}\text { 各相同性参 } \\
\text { 数(A) }\end{array}$ & $Q_{s a}=480, \quad b=10$ \\
\hline 基本参数 $(\mathrm{F})$ & $M=11, E=251.7 \mathrm{GPa}, v=0.33, Q_{0}=411.9 \mathrm{MPa}$ \\
\hline $\begin{array}{c}\text { 随动硬化参 } \\
\text { 数 }(\mathrm{F})\end{array}$ & $\begin{array}{l}\zeta_{1}=639.0, \zeta_{2}=197.4, \zeta_{3}=114.5, \zeta_{4}=69.6, \zeta_{5}=49.8 \\
\zeta_{6}=38.7, \zeta_{7}=31.6, \zeta_{8}=26.7, \zeta_{9}=18.9, \zeta_{10}=10.4, \zeta_{11}=2.1, \\
r_{1}=35.7, r_{2}=62.5, r_{3}=52.8, r_{4}=52.7, r_{5}=352, r_{6}=37.7 \\
r_{7}=27.9, r_{8}=41.4, r_{9}=62.7, r_{10}=21.9, r_{11}=2029.6\end{array}$ \\
\hline $\begin{array}{c}\text { 各相同性参 } \\
\text { 数(F) }\end{array}$ & $Q_{s a}=580, \quad b=30$ \\
\hline
\end{tabular}

3.3.3 循环弹塑性本构模型参数综合验证结果

为验证第 3.1 节所介绍的循环本构模型和第 3.3 节中表征所得参数对双相不锈钢中奥氏体相和铁素 体相循环变形行为的合理性和适用性, 本节首先对 该本构模型进行 ABAQUS 有限元移植, 后采用表 2 中表征所得参数, 结合图 8 中的有限元模型, 分别 模拟奥氏体相和铁素体相在不同循环载荷水平下 (加载峰值载荷分别为 $50 \mathrm{mN} 、 100 \mathrm{mN}$ 和 $200 \mathrm{mN}$, 谷值载荷为 $5 \mathrm{mN}$ ) 的纳米压痕过程和循环变形行 为。模拟和试验所得的奥氏体相和铁素体相的接触 载荷-压入深度演化曲线和峰值压入深度-循环周次 演化曲线如图 $6 \mathrm{~b} 、 6 \mathrm{c}$ 所示。可以看出, 模拟结果与 试验结果非常接近。由于材料的压痕棘轮行为是一 种二次变形, 与宏观棘轮类似, 实际应用中更加关 注棘轮变形随循环周次的演化。此处对图 6c 的试验
结果和模拟结果进行误差分析，得到的奥氏体和铁 素体压痕棘轮演化曲线的平均相对误差如表 3 所 示。当最大接触载荷为 $50 \mathrm{mN} 、 100 \mathrm{mN}$ 和 $200 \mathrm{mN}$ 时，在大部分工况下奥氏体和铁素体的平均相对误 差均小于或接近 5\%。鉴于对二次变形的描述能力, 第 3.1 节介绍的循环弹塑性本构模型和本文关于循 环塑性参数的表征方法在描述双相不锈钢各组相的 循环压痕行为时合理可用。

表 3 压痕棘轮模拟值和预测值的相关系数

\begin{tabular}{cccc}
\hline & \multicolumn{3}{c}{ 载荷水平 $/ \mathrm{mN}$} \\
\cline { 2 - 4 } 组相 & 50 & 100 & 200 \\
\hline 奥氏体相 $(\%)$ & 3.5 & 3.7 & 4.8 \\
铁素体相 $(\%)$ & 6.0 & 5.7 & 4.1 \\
\hline
\end{tabular}

为进一步验证考虑循环硬化之后的循环本构模 型和参数对单向压痕过程的适用性, 又采用表 2 的 参数对两相的单向压痕过程进行有限元模拟。模拟 和试验所得的接触载荷-压入深度演化曲线如图 11 所示，根据式(15)求得的奥氏体和铁素体最大压入 载荷模拟值和试验值的相对误差约为 $3.9 \%$ 和 $0.1 \%$, 可见模拟与试验结果吻合度非常好。综上所述，根 据压痕试验结果和基于修正的 ABDEL-KARIMOHNO 非线性随动硬化演化准则的弹塑性本构模 型, 建立的塑性和循环塑性参数表征方法合理可用。

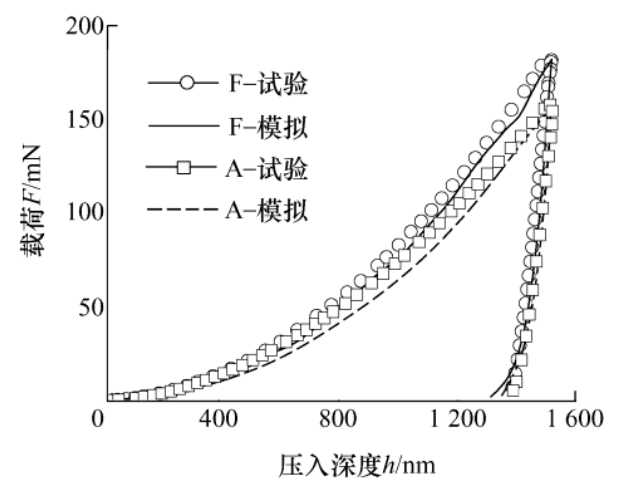

图 11 奥氏体相和铁素体相的接触载荷-压入深度对比曲线

\section{4 双相不锈钢变形行为的有限元分析}

前文通过两相单向和循环纳米压痕的模拟和对 比验证了本文所选弹塑性有限元本构模型及表征参 数的合理性。本节将基于对第 3.1 节介绍的循环弹 塑性本构模型的有限元移植和扩展应用，采用代表 性微结构体积单元，模拟获取双相不锈钢的宏观拉 伸应力-应变曲线和应力控制循环下的棘轮行为。最 终，与第 1.2 节所介绍的宏观试验结果进行对比和 分析, 进一步验证上述本构模型和参数对宏观材料 


\section{的适用性。}

\section{1 有限元模型}

首先，根据图 1 中计算出的双相不锈钢中奥氏 体相和铁素体相的体积占比, 采用 V-T(VORONOI TESSELLATION)法生成具有不同晶粒分布特征的 代表性微结构体积单元, 如图 12a、12b 所示(其中 浅灰色区域代表奥氏体相, 深灰色区域代表铁素体 相, 两种晶粒尺寸均与实际晶粒尺寸相当)。之后, 采用四边形平面应力单元对具有两种分布特征的代 表性微结构体积单元进行网格划分。同时, 考虑材 料的周期性, 对代表性单元的上侧和下侧分别施加 简化的周期性边界条件, 对其左侧施加法向位移约 束, 右侧施加法向位移或循环载荷。有限元模拟时 采用第 3.1 节介绍的循环弹塑性本构模型, 分别对 奥氏体相和铁素体相赋予第 3.3 节表 2 所列的塑性 和循环塑性相关参数。

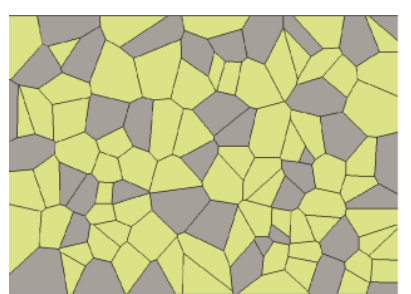

(a) 模型 1

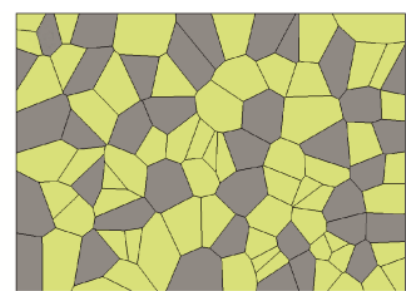

(b) 模型 2
图 12 双相不锈钢微结构模型

\section{2 双相不锈钢单轴拉伸行为的有限元分析}

为验证本文选取的本构模型及表征参数对双相 不锈钢宏观拉伸行为的适用情况, 本节中利用上述 的 2 个有限元模型对双相不锈钢的单轴拉伸过程进 行模拟, 最终通过体积加权平均方法获得双相不锈 钢的平均应力-应变曲线。模拟结果与第 1.2 节中单 轴拉伸试验曲线的对比如图 13 所示。可以看出, 模 拟所得的双相不锈钢宏观拉伸应力-应变曲线与试 验结果吻合很好。

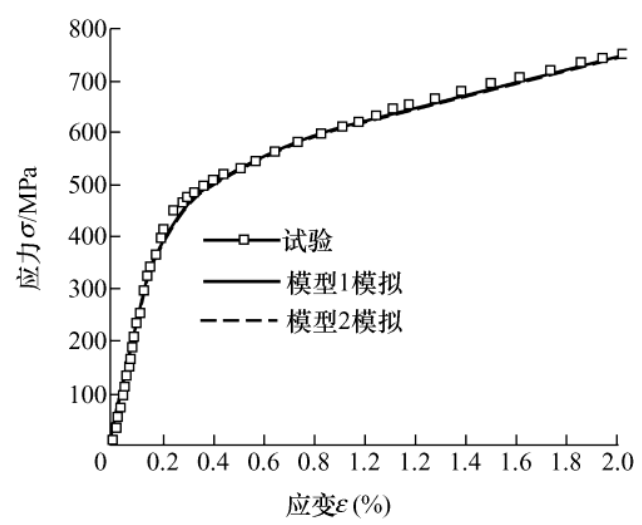

图 13 双相不锈钢试验单向拉伸曲线与模拟结果对比

\section{3 双相不锈钢循环变形行为的有限元分析}

为验证本文的本构模型及表征参数对双相不 锈钢宏观循环变形行为的适用情况，本节仍利用 第 4.1 节中的有限元模型及约束方式，对代表性 体积单元的右侧施加循环应力载荷, 模拟双相不 锈钢在循环应力载荷下的棘轮行为。根据第 1.2 节中双相不锈钢的宏观循环变形试验, 在模拟过

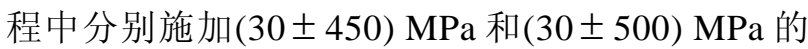
循环应力载荷, 设置循环周次为 40 周。模拟完成 后通过体积加权平均方法获得双相不锈钢棘轮应 变随循环周次的演化曲线 $\left(\varepsilon_{r}=\left(\varepsilon_{\max }+\varepsilon_{\mathrm{min}}\right) / 2\right)$, 所得 模拟曲线和相应的试验曲线如图 14 所示。可以看 出, 模拟结果和试验结果从数值和演化趋势上都 非常接近。对试验结果和模拟结果进行误差分析, 得到双相不锈钢棘轮演化曲线的平均相对误差,

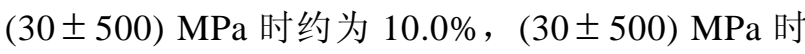
约为 $8.5 \%$ 。对双相不锈钢棘轮行为的模拟误差略 高于对奥氏体相、铁素体相和双相不锈钢宏观拉 伸行为的模拟结果。但由于材料棘轮行为是一种 复杂的二次变形, 且参数确定需要同时兼顾材料 单调加载和循环加载变形两种情况。从对二次变 形描述来看，上述结果合理。

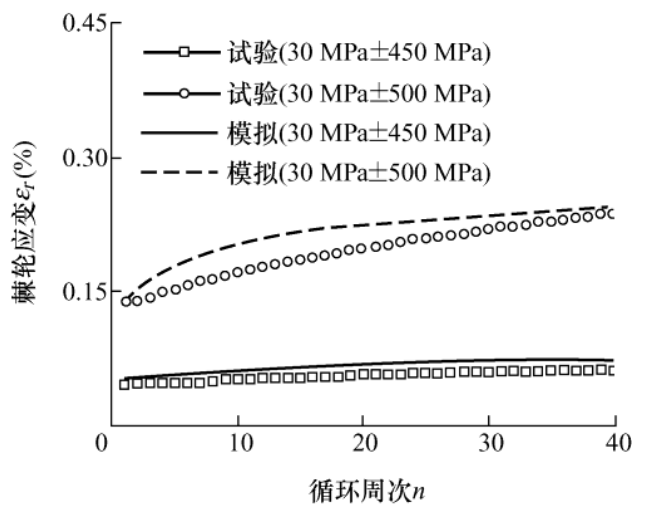

图 14 双相不锈钢不同载荷条件下的棘轮演化曲线

综合第 3 节和第 4 节的模拟和验证, 可见本 文选取的基于修正的 ABDEL-KARIM-OHNO 非 线性随动硬化演化准则的弹塑性本构模型, 结合 新发展的基于纳米压痕试验的材料塑性和循环塑 性参数表征方法, 可以合理实现对双相材料从微 观到宏观的变形和循环变形描述。新发展的参数 标定方法，不仅适用于对材料微观纳米压痕过程 (单向+循环)的准确模拟分析, 还适用于对宏观材 料的单轴拉伸和循环变形行为的模拟和描述。相 关研究对获取多相材料的局部微观力学性能和无 法大尺寸取样材料的宏观力学性能提供了有效方 法和手段。 


\section{5 结论}

(1) 双相不锈钢中铁素体相材料强度总体高于 奥氏体相, 两者通过晶界相联系并存在交互作用, 奥氏体中距离晶界越远, 局部材料强度越弱, 铁素 体中距离晶界越远，局部材料强度越强。随着距离 的增加, 晶界对两相组织产生的影响变小。

(2) 奥氏体相与铁素体相均有明显的压痕棘轮 现象, 载荷水平越高现象越明显, 并在前 $3 \sim 5$ 循环 周内压入深度快速增长, 然后逐步减小。在相同的 载荷水平下, 铁素体相的压入深度远低于奥氏体相 的压入深度, 铁素体的抗棘轮变形能力高于奥氏体。

(3) 基于对双相不锈钢中奥氏体相和铁素体相 单向和循环纳米压痕试验的有限元反演和分析, 本 文发展了一套材料塑性和循环塑性参数表征方法。 该方法所获得的参数对模拟双相不锈钢各组相的微 观循环纳米压痕行为和宏观拉伸、循环变形行为均 合理可用。

\section{参 考 文 献}

[1] WANG Xu, WANG Jianquan, WANG Jianbiao, et al. Anomalous sudden drop of temperature-dependent Young's modulus of a plastically deformed duplex stainless steel[J]. Materials \& Design, 2019, 181(5): 71-80.

[2] 于海成, 严与辉, 刘小杨, 等. 双相不锈钢成分、性能 及析出相分析 [J]. 特殊钢, 2019, 40(3)：53-58.

YU Haicheng, YAN Yuhui, LIU Xiaoyang, et al. Analysis on composition, properties and precipitated phases of duplex stainless steel[J]. Special Steel, 2019, 40(3): $53-58$.

[3] ZHANG Fan, SUN Pengfei, LI Xiaocui, et al. A comparative study on microplastic deformation behavior in a $\mathrm{SiCp} / 2024 \mathrm{Al}$ composite and its unreinforced matrix alloy[J]. Materials Letters, 2001， 49(2): 69-74.

[4] ZHU Mingliang, XUAN Fuzhen. Effect of microstructure on strain hardening and strength distributions along a Cr-Ni-Mo-V steel welded joint[J]. Materials \& Design, 2015, 65: 707-715.

[5] 徐连勇, 张舒婷, 荆洪阳, 等. $\mathrm{Ag}-\mathrm{GNSs} / \mathrm{SnAgCu}$ 钎料 纳米压痕变形行为研究 [J]. 机械工程学报, 2018, 54(8): 151-156.

XU Lianyong, ZHANG Shuting, JING Hongyang, et al. Research on the deformation behavior of $\mathrm{Ag}-\mathrm{GNSs} / \mathrm{SnAgCu}$ solders during nanoindentation tests[J]. Journal of Mechanical Engineering, 2018， 54(8): 151-156.

[6] NILSSON J O. Super duplex stainless steels[J]. Materials Science and Technology, 2013, 8(8): 685-700.

[7] 谢季佳, 洪友士. 纳米晶 $\mathrm{Ni}$ 疲劳行为的试验研究 [J]. 金 属学报, 2009, 45(7): 844-848.

XIE Jijia, HONG Youshi. Experimental investigation on fatigue behavior of nanocrystalline nickel[J]. Acta Metallurgica Sinica, 2009, 45(7): 844-848.

[8] JIA Yunfei, XUAN Fuzhen, YANG Fuqian. Viscoplastic response of tooth enamel under cyclic microindentation[J]. Materials Science and Engineering: C, 2015, 55: 448-456.

[9] YANG Fuqian, PENG Lingling, OKAZAKI K. Cyclic indentation in aluminum[J]. Journal of Materials Science, 2007, 42(12): 4513-4520.

[10] CUI Yuanyuan, JIA Yunfei , XUAN Fuzhen. Micro-deformation evolutions of the constituent phases in duplex stainless steel during cyclic nanoindentation[J]. Scientific Reports，2018，8(1): 6199-6208.

[11] 孔祥霞, 孙风莲, 杨氷森, 等. $\mathrm{Bi}$ 和 $\mathrm{Ni}$ 元素对 $\mathrm{Cu} / \mathrm{SAC} / \mathrm{Cu}$ 微焊点体钎料蠕变性能的影响 [J]. 机械工程学报, 2017, 53(2): 53-60.

KONG Xiangxia, SUN Fenglian, YANG Miaosen, et al. Effect of $\mathrm{Bi}$ and $\mathrm{Ni}$ concentration on the creep behavior of the bulks of $\mathrm{Cu} / \mathrm{SAC} / \mathrm{Cu}$ micro solder joints[J]. Journal of Mechanical Engineering, 2017，53(2): 53-60.

[12] DAO M, CHOLlaCOOP N, VAN V, et al. Computational modeling of the forward and reverse problems in instrumented sharp indentation $[\mathrm{J}]$. Acta Materialia, 2001, 49(19): 3899-3918.

[13] PELLETIER H. Predictive model to estimate the stress-strain curves of bulk metals using nanoindentation[J]. Tribology International, 2006，39(7): 593-606.

[14] 马永, 姚晓红, 田林海, 等. 利用纳米压入的反演分析 法确定金属材料的塑性性能 [J]. 金属学报, 2011, 47(3): 321-326.

MA Yong, YAO Xiaohong, TIAN Linhai, et al. Extract the plastic properties of metals using reverse analysis of nanoindentation test $[\mathrm{J}]$. Acta Metallurgica Sinica, 2011, 47(3): 321-326

[15] HAN H N, LEE C G, OH C S, et al. A model for deformation behavior and mechanically induced martensitic transformation of metastable austenitic steel[J]. Acta Materialia, 2004, 52(17): 5203-5214.

[16] PIERCE D T, JIMÉNEZ J A, BENTLEY J, et al. The 
influence of stacking fault energy on the microstructural and strain-hardening evolution of Fe-Mn-Al-Si steels during tensile deformation[J]. Acta Materialia, 2015, 100: 178-190.

[17] CHOI J Y, LEE J, LEE K, et al. Effects of the strain rate on the tensile properties of a TRIP-aided duplex stainless steel[J]. Materials Science and Engineering A, 2016, 666: 280-287.

[18] KANG Guozheng, GAO Qing, YANG Xianjie. A visco-plastic constitutive model incorporated with cyclic hardening for uniaxial/multiaxial ratcheting of SS304 stainless steel at room temperature[J]. Mechanics of Materials, 2002, 34: 521-531.

作者简介: 郭素娟, 女, 1982 年出生, 博士。主要研究方向为过程设备 结构完整性。

E-mail: sujuanguo@ecust.edu.cn

赵剑(通信作者), 男, 1984 年出生, 博士, 助理教授。主要研究方向为 复合材料与非均匀材料和结构的有限元分析和试验验证。

E-mail: zhaojian@tongji.edu.cn 